\title{
CASEFILE
}

\section{A Doppler-Cancellation Technique for Determining the Altitude Dependence of Gravitational Red Shift in an Earth Satellite*}

\author{
R. S. BADESSA $\dagger$, R. L. KENT $\dagger$, Member, IRE, J. C. NOWELL $\ddagger$, AND C. L. SEARLE $\dagger$, MEMbER, IRE
}

Summary-A cancellation technique permits measurement of the frequency of a source moving relative to an observer without the obscuring effect of first-order Doppler shifts. The application of this method to a gravitational red shift experiment involving the use of an earth satellite containing a highly stable oscillator is described. The rapidity with which a measurement can be made permits the taking of data at various altitudes in a given elliptical orbit. Tropospheric and ionospheric effects upon the accuracy of results are estimated.

\section{INTRODUCTION}

$\AA^{\mathrm{CCO}}$ CCORDING to the General Theory of Relativity, the time kept by a "proper" clock (or the frequency of a standard oscillator) is affected by the motion of the clock and by its local gravitational potential. The latter effect, known as the "gravitational red shift," is expected to result in variations of only 7 parts in $10^{10}$ in the rates of clocks in the vicinity of the earth. Attempts to obtain experimental verification have been

* Original manuscript received by the IRE, November 23, 1959. This work was supported in part by the National Aeronautics and Space Administration under Contract NASw-33.

† Res. Lab. of Electronics, Mass. Inst. Tech., Cambridge, Mass.

‡ Missile Systems Div., Raytheon Manufacturing Co., Waltham Mass. Formerly with Res. Lab. of Electronics, Mass. Inst. Tech., Cambridge, Mass. stimulated by the development of highly stable frequency standards and the availability of earth satellites in orbits that provide a significant variation in gravitational potential.

In 1956, Singer ${ }^{1}$ proposed the measurement of gravitational red shift by an experiment involving comparison of the time kept by a stable clock in a satellite with that of an identical clock on earth. Since then, several versions of his experiment have been suggested. Basically, all of them involve the use of an atomic clock, or equivalent standard, from which, by frequency division, a series of precise timing pulses can be derived. These pulses would be transmitted as a modulated carrier over a one-way link from a satellite to earth, where they would be compared with a similar, locally generated timing waveform to determine the frequency difference. The frequency of the received pulses is directly influenced by the rate-of-change of path lengths (firstorder or radial Doppler shift). This effect is typically four or five orders of magnitude larger than the gravita-

${ }^{1}$ S. F. Singer, "Application of an artificial satellite to the measurement of the general relativistic 'red shift'," Phys. Rev., vol. 104, pp. 11-14; October, 1956. 
tional red shift. Consequently, data must be, acquired
over a sufficiently long time interval (probably several days) to insure that path-length measurement errors are negligible. At the completion of this experiment, a single value is obtained for the average frequency shift over many orbital periods. Thus it is not possible by this technique to determine the effect of altitude variations.

In order to obtain the altitude dependence of gravitational red shift, the measurement interval must be shortened to a small fraction of the orbital period. However, path-length variations make the performance of a short-time measurement in a one-way propagation experiment virtually impossible. But two-way propagation experiments offer definite possibilities. Engineers are quite familiar with the doubling of the first-order Doppler shift, which occurs in transmission of signals to and from a radar target. If it were possible to reverse the sense (i.e., direction) of one of these frequency shifts by a mixing operation, then essentially complete cancellation of first-order Doppler shift could be effected. In the short-time measurement system ${ }^{2}$ that will be described, such a technique permits data for a single altitude to be obtained in an interval of 60 seconds or less. The following significant advantages result.

1) By employing an elliptical orbit, frequency differences at various altitudes can be observed, and hence the variation of frequency with gravitational potential can be obtained.

2) If the measurement is repeated when the satellite is at a particular altitude (e.g., at perigee), a slow frequency drift between satellite and earth clocks can be determined and removed. Since the quantity of interest is the variation in frequency difference as a function of altitude, a fixed offset frequency between clocks is inconsequential. Therefore, quartz-crystal oscillators can probably be employed.

3) Continuous integration or counting of pulses over many orbital periods is not required. Skipped cycles or temporary failures would not invalidate the experimental results.

\section{The Short-Time Measurement Principle}

The principle employed in a short-time measurement will be illustrated by a simplified analysis. In the interest of simplicity, the measurements are assumed to take place at an earth pole ( $O$ in Fig. 1). (An outline of the derivation of the following expressions is given in the Appendix.)

\footnotetext{
2 R. S. Badessa, R. L. Kent, and J. C. Nowell, "Short-time meas-
} urement of time dilation in an earth satellite," Phys. Req. Lett.,
vol. 3, pp. 79-80; July, 1959.

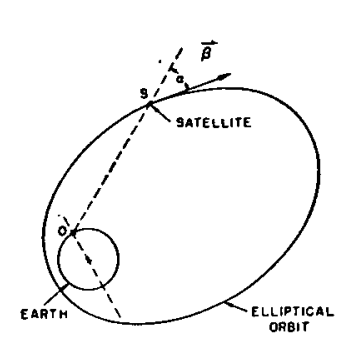

Fig. 1-Geometry of the measurement problem.

1) A transmitter (located at point $O$ ) of frequency $f$ transmits to the satellite.

2) The frequency of the signal received at the satellite is

$$
f^{\prime}=f \frac{1-\beta \cos \alpha}{\left(1-\beta^{2}+2 \phi\right)^{1 / 2}}
$$

where $\beta$ is the magnitude of the satellite velocity (measured in the coordinate system of the observer at $O$ ) at the instant of reception, normalized in terms of the velocity of light; $\alpha$ is the angle (measured in the same coordinate system) between the propagation path $O-S$ and the velocity vector $\vec{\beta}$; $\phi$ is the (positive) difference in gravitational potential between points $S$ and $O$, normalized in terms of the square of the velocity of light.

3) The satellite contains an oscillator of frequency $2 f$ (measured on the satellite). The received signal frequency $f^{\prime}$ is subtracted from $2 f$ by mixing within the satellite to yield a frequency difference

$$
f^{\prime \prime}=2 f-f^{\prime}
$$

4) The mixture signal $f^{\prime \prime}$ is transmitted back to earth. (Reception and retransmission at the satellite are assumed to be virtually instantaneous.)

The frequency of the received signal is measured as

$$
f^{\prime \prime \prime}=f^{\prime \prime} \frac{\left(1-\beta^{2}+2 \phi\right)^{1 / 2}}{1+\beta \cos \alpha} .
$$

Substitution for $f^{\prime \prime}$ in (3) yields

$$
f^{\prime \prime \prime}=f\left[2-\frac{1-\beta \cos \alpha}{\left(1-\beta^{2}+2 \phi\right)^{1 / 2}}\right]\left[\frac{\left(1-\beta^{2}+2 \phi\right)^{1 / 2}}{1+\beta \cos \alpha}\right] .
$$

By means of a power-series expansion, retaining secondorder terms in $\beta$ and first-order terms in $\phi$, we obtain

$$
f^{\prime \prime \prime} \approx f\left(1+2 \phi-\beta^{2}\right) .
$$

The first-order Doppler term has been cancelled, and the received signal frequency differs from the ground transmitter frequency by

$$
\Delta f \approx f\left(2 \phi-\beta^{2}\right) .
$$


The two terms in (5) are apparently inseparable experimentally. The first term, $2 \phi f$, is the desired gravitational frequency shift. The second term, $-\beta^{2} f$, is the wellknown second-order, or transverse, Doppler shift of special relativity. This term, which has been verified experimentally, can be calculated easily and subtracted from the measured frequency difference to obtain the residual gravitational effect.

The frequency difference in the general case of an observer not located at the pole (Fig. 2) is given by

$$
\begin{array}{r}
\Delta f=f\left[2 \phi-\left(\beta_{s}{ }^{2}-\beta_{0}{ }^{2}\right)-2 \beta_{0} \beta_{s} \cos \left(\alpha_{2}+\gamma_{2}\right)\right. \\
\left.-\beta_{0}{ }^{2}\left(1+r \sin \gamma_{2}\right)\right],
\end{array}
$$

where $\beta_{s}, \beta_{0}, \alpha_{2}$, and $\gamma_{2}$ are as shown in Fig. 2, measured in a nonrotating coordinate system with origin at the earth's center and $r$ is the range, normalized in terms of the earth's radius. The term in (6a) containing the product $\beta_{0} \beta_{8}$ is of the order of $10^{-10}$, and cannot be neglected in the present experiment. This term must be retained, and appropriate values substituted. Present orbital computational accuracy is more than adequate for this purpose.

\section{Satellite Equipment}

As we have indicated, the operations to be performed in the satellite are as follows:

1) Amplify a received signal of frequency $f^{\prime}$.

2) Mix the received signal with that of the satellite "clock" to obtain $2 f-f^{\prime}$.

3) Amplify and transmit a signal of frequency $2 f-f^{\prime}$.

In order for the cancellation of first-order Doppler shifts to be effective, the signals received and transmitted at the satellite must be very nearly of the same frequency. One way of avoiding the ensuing "spillover" problem is to employ pulsed waveforms. The received signal pulses, $1-10 \mu \mathrm{sec}$ long, are stored briefly in a delay line or equivalent device. After the reception of each pulse, the receiver is turned off and the transmitter turned on. Fig. 3 is a block diagram of the satellite equipment required to instrument this technique. This configuration is intended only for purposes of illustration. An operating frequency range of $500-1000 \mathrm{mc}$, which is expected to fall between regions of severe ionospheric and tropospheric propagation anomalies, has been tentatively selected. A factor in the final choice of an operating frequency is the desirability of employing transistor circuitry. For ranges up to 10,000 miles, an RF output of approximately $20 \mathrm{mw}$ (average), 0.4 watt (peak) is required from the satellite transmitter.

Although duplication of units is not indicated in the block diagram, it is particularly desirable to include more than one crystal frequency standard in the satellite, to permit independent measurements and to improve system reliability. The use of three crystal oscillators in a common temperature-stabilized oven is suggested. The crystals, which could be offset from one another in frequency for identification purposes, might

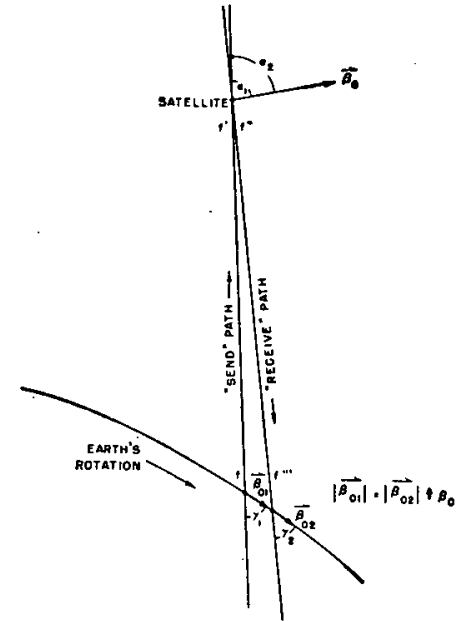

Fig. 2-Geometry of the two-way propagation experiment.

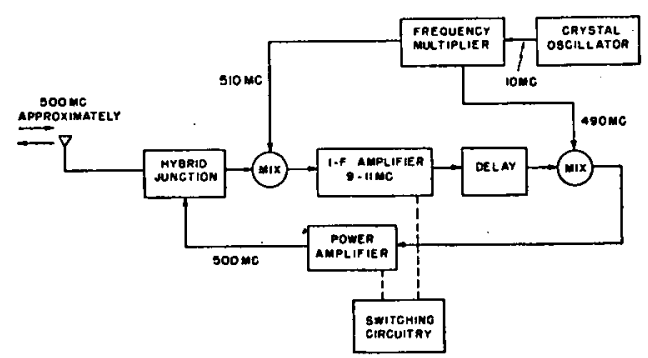

Fig. 3-Illustrative satellite-equipment configuration.

be selected with different known temperature coefficients. Then variations in satellite temperature, which could possess a deceiving similarity to the orbital period of the satellite itself, will affect the three oscillators in varying degrees. Thus, a means for separating relativistic frequency shifts from temperature-dependent effects is provided. Preliminary power and weight estimates for the satellite equipment, including the weight estimate for batteries, are 3 watts and 35 pounds.

The stability of the crystal oscillators is of paramount importance and will be discussed separately. The overall phase shift through the receiver and transmitter should remain stable within $30^{\circ}$ over the measurement interval 20-60 seconds, in order not to degrade the measurement accuracy which should be determined essentially by the short-term stability of the crystal oscillators.

\section{Ground Equipment}

An illustrative block diagram of the major portion of the required ground equipment is shown in Fig. 4. It functions as follows:

1) A signal of frequency $f$ is transmitted.

2) A signal of frequency $f^{\prime \prime \prime}$ is received from the satellite and compared in frequency with $f$ by means of a mixer and a synchronous detector.

3) The frequency-difference signal is recorded on tape together with a fixed-frequency reference signal. 
The transmitted and received signals both consist of series of pulses (each 1-10 $\mu$ sec long). Time-delay discrimination is provided in the receiver for the rejection of transmitter feed-through signals and other undesired signals. In addition, a frequency offset of 1 part in $10^{6}$ is purposely introduced between the ground and satellite frequency standards. This insures a small frequency separation between the signals transmitted and received on the ground, thereby facilitating resolution of the satellite signal from clutter by means of narrow-band filtering, without materially affecting first-order Doppler cancellation.

The coherent waveform and narrow-band filtering $( \pm 1 \mathrm{cps})$ employed in the ground receiver make possible the low requirement on satellite transmitter power (20 $\mathrm{mw}$, average). Doppler tracking is unnecessary because the frequency of the signal received on the ground is virtually independent of first-order Doppler shift. However, the first-order Doppler shift is present in the signal received on the satellite. For simplicity, broad-band amplification, rather than Doppler tracking, is employed there, at the expense of increased groundtransmitter power. For a 10 meter $^{2}$ antenna on the ground, the power required to yield a clean signal on the satellite is approximately 500 watts (average), $10 \mathrm{kw}$ (peak).

The ground station requires means for storage, as well as immediate processing, of the receiver output signal. The desired information is contained in the small variations in output frequency with orbital position. Since orbital position will be known as a function of time, a recording of the output signal together with a time signal will suffice.

Processing of the data involves measurement of a small frequency difference between two signals. This is best accomplished by measuring the total phase difference accumulated in a specific time interval. For example, a frequency difference of $0.1 \mathrm{cps}$ in a 60 -second interval results in a total accumulated phase difference of $2160^{\circ}$. This can be measured precisely by manual phase-tracking techniques, with the use of an oscilloscope as a visual tracking indicator.

\section{Oscillator Stability}

The frequency standards used in this experiment must meet two separate stability requirements. First, all random and periodic frequency excursions must be at least an order of magnitude smaller than the total effect that is to be measured. Second, any long-term frequency drift in one orbital period should not exceed 20 to 30 per cent of the total desired effect. Perhaps the reasons for these stability requirements can best be illustrated by plotting a set of hypothetical data that might be obtained in this experiment. Fig. 5 shows a plot of the hypothetical frequency difference against time. For simplicity, it is assumed that data are acquired alternately at apogee and at perigee for several successive sightings. The clusters of points at times $A, B, C$, and so

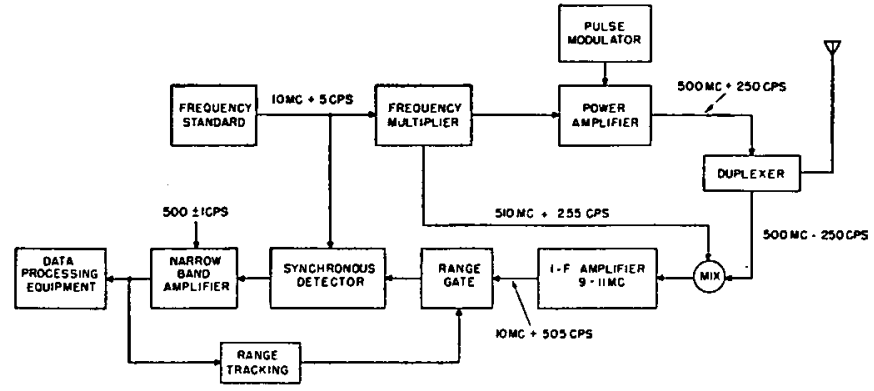

Fig. 4-Illustrative ground-equipment configuration.

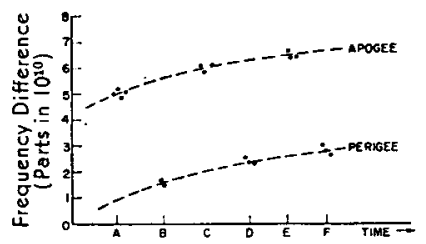

Fig. 5-Hypothetical data to illustrate oscillator stability requirements.

on, represent several readings of frequency difference obtained during the intervals when the satellite is visible. The frequency difference between the two dashed lines for apogee and perigee readings is expected to be approximately 4 parts in $10^{10}$. Therefore, the undesired random and periodic frequency variations of the oscillators that are indicated by the dispersion of the clusters must be no greater than a few parts in $10^{11}$.

The slope of either of the dashed lines in Fig. 5 represents the difference between the long-term drifts of the oscillators, and it is clear that a monotonic drift per orbital period of from 20 to 30 per cent of the total effect can easily be tolerated. For the proposed experiment, a drift of 1 part in $10^{10}$ in a few hours, or approximately 1 part in $10^{9}$ per day, is permissible. A small fixed offset frequency between the oscillators would merely shift the base line in Fig. 5, and therefore would have no effect upon the desired measurement.

Actual measurements taken on commercially available quartz-crystal oscillators indicate that these oscillators essentially meet the stability requirements. Table I shows some data obtained in a comparison test. The 1-mc output signals of two crystal oscillators were multiplied up and mixed down in such a way as to magnify their frequency difference by a factor of 1000 . The resulting beat frequency was then determined by means of a counter operating over periods of 10 and 100 seconds. The last digit of the counter output represents parts in $10^{10}$ for the 10 -second count, and parts in $10^{11}$ for the 100 -second count. In either case, the counter has a starting error of one count. Thus, the differences indicated between the two oscillators may be entirely accounted for by the counter error. The oscillator error is shown to be no greater than 1 part in $10^{11}$ when it is averaged over successive 100 -second intervals. 
TABLE I

Frequency Comparison Data for Two Hermes Model 101c 1-MC Crystal Frequency Standards

\begin{tabular}{c|c}
\hline $\begin{array}{c}\text { 10-Second Counting Interval; } \\
\text { Total Elapsed Time, } \\
\text { Approximately 45 Minutes. }\end{array}$ & $\begin{array}{c}\text { 100-Second Counting Interval; } \\
\text { Total Elapsed Time, } \\
\text { Approximately 15 Minutes }\end{array}$ \\
\hline 93101 & 31005 \\
93100 & 31006 \\
93100 & 31006 \\
93100 & 31005 \\
93100 & 31006 \\
$:$ & 31006 \\
$:$ & 31006 \\
93100 & \\
93100 & \\
93101 & \\
93100 & \\
93101 & \\
\hline
\end{tabular}

The oscillators that were tested employed vacuumtube circuits; data are not available for a transistor version that is now being developed. However, the stability is believed to be determined primarily by the crystal and its oven, and not by the oscillator circuitry. Therefore no degradation in stability is expected to result from a change to transistors.

In order to transfer the oscillator stability to a transmitter operating in the UHF range, a chain of frequency multipliers is required. Two methods of frequency multiplication, both of which use variable-capacitance diodes as the multiplying elements, are being considered. Leeson and Weinreb, ${ }^{3}$ in a detailed study of low-order frequency multipliers with nonlinear reactances, demonstrated that frequency doublers with only 1-db loss could be constructed. A chain of doublers that are now being developed to go from the oscillator frequency to the transmitter frequency is expected to yield a loss of only $10-15 \mathrm{db}$. The second approach to the frequencymultiplication problems is the use of one or two large frequency jumps. However, experimental results thus far indicate that in multiplying by a factor of 100 , the loss is approximately $40 \mathrm{db}$.

\section{Propagation Effects}

As we have stated, the success of the described measurement depends upon the cancellation of the first-order Doppler shift, an effect that is four or five orders of magnitude greater than the gravitational red shift. For adequate cancellation, it is essential that random fluctuations in the effective path lengths upward and downward be small, or that they be well correlated. Phase fluctuations along the propagation paths are known to be caused by turbulent irregularities in the troposphere and in the $\mathrm{F}_{2}$ region of the ionosphere. Extensive dis-

${ }^{3}$ D. B. Leeson and S. Weinreb, "Frequency multiplication with nonlinear capaciturs-a circuit analysis," Proc. IRE, vol. 47, p 2076-2084; December, 1959. cussions of these and related effects have been published. ${ }^{4-10}$

It is desirable to select an operating frequency in a region in which the total rms phase error that is expected to arise from propagation anomalies falls well within the total permissible phase error of the experiment. If the frequency shift is computed by observing the total accumulated phase difference in a specified time interval $T$, then the resulting frequency error cannot exceed $\Delta \psi / \pi T$ cps, where $\Delta \psi$ is the total uncancelled phase fluctuation. At $500 \mathrm{mc}$, an rms phase error of 0.5 radian results in a 1 per cent error in frequency for a 60-second measurement interval. Such an error would be acceptable, since it is less than the value of the expected oscillator instability.

Tropospheric fine structure is measured by variations in the dielectric constant. The rms one-way phase fluctuation obtained by Muchmore and Wheelon ${ }^{4}$ is given by

$$
\psi_{\mathrm{rms}} \approx \frac{\left(2 l_{0} L\right)^{1 / 2} \pi \Delta e}{\lambda}, \quad \text { for } L \gg l_{0}
$$

where $l_{0}$ represents the scale of the irregularities (probably 200 feet); $L$ is the propagation path length through the turbulent medium (taken to be 20,000 feet); $\Delta e$ is the rms deviation of the dielectric constant from unity (probably $10^{-6}$ ); and $\lambda$ is the wavelength. At a frequency of $1000 \mathrm{mc}$, the one-way phase fluctuation is computed as approximately $0.6^{\circ} \mathrm{rms}$. This value is believed to be representative for a turbulent mass of (optically) clear air. In the presence of rain clouds, the interiors of which are very turbulent and contain large fluctuations in dielectric constant, the situation is somewhat different. If we take as representative values for a rain cloud $l_{0}=20$ feet, $L=5000$ feet, $\Delta e=20 \times 10^{-6}$, the one-way fluctuation in phase at $1000 \mathrm{mc}$ is found to be approximately $1.6^{\circ} \mathrm{rms}$. Thus, a net phase fluctuation of $2^{\circ}$ or $3^{\circ} \mathrm{rms}$ might be typical for $1000-\mathrm{mc}$ one-way propagation through the troposphere on a cloudy day.

The tropospheric phase-fluctuation measurements of Herbstreit and Thompson, ${ }^{10}$ at $1046 \mathrm{mc}$, appear to be in

- R. B. Muchmore and A. D. Wheelon, "Line-of-sight propagation phenomena. I. Ray treatment," Proc. IRE, vol. 43, pp. 1437-1449; October, 1955.

5J. L. Pawsey and R. N. Bracewell, "Radio Astronomy," Clarendon Press, Oxford, Eng., pp. 347-351; 1955.

${ }^{\circ} \mathrm{H}$. G. Booker, "The use of radio stars to study" irregular refraction of radio waves in the ionosphere," Proc. IRE, vol. 46, pp. 298314; January, 1958.

7 D. F. Martyn, "The normal F region of the ionosphere," Proc. IRE, vol. 47, pp. 147-155; February, 1959.

'C. O. Hines, "Motion in the ionosphere," Proc. IRE, vol. 47, pp. 176-186; February, 1959.

9 "Propagation Factors Affecting Long-Range UHF Radars at High Latitudes," Defence Res. Telecommun. Establ., Ottawa, Ont., Can., Rept. No. 41-1-3; December, 1958.

${ }_{10} \mathrm{~J}$. W. Herbstreit and M. E. Thompson, "Measurements of the phase of radio waves received over transmission paths with electrical lengths varying as a result of atmospheric turbulence," PROC. IRE, vol. 43, pp. 1391-1401; October, 1955. 
general agreement with theory. The standard deviation of the phase shift over a 10-mile tropospheric path exceeded $3^{\circ}$ fifty per cent of the time, and $5.5^{\circ}$ ten per cent of the time. Moreover, the fluctuation period was found to be approximately 1 hour for large fluctuations, with minute-to-minute variations in phase shift of only $0.3^{\circ}$ rms. Since the tropospheric phase fluctuation, from (6b), varies directly with the operating frequency, we conclude that the contribution of the troposphere to measurement errors in this experiment is negligible.

Ionospheric fluctuations are measured by variations in the free-electron density. Turbulent irregularities in the $F_{2}$ region are maximum near midnight (local time) and occur in a region roughly $55 \mathrm{~km}$ thick at a nighttime altitude of approximately $300 \mathrm{~km}$. The one-way rms phase fluctuation is shown by Booker ${ }^{6}$ to be

$$
\psi_{\mathrm{rms}}=\left(\frac{h_{0} B}{2}\right)^{1 / 2} r_{e} \lambda \Delta N
$$

where $h_{0}, H$ are analogous to $l_{0}, L$ for the troposphere (taken here to be $1 \mathrm{~km}$ and $55 \mathrm{~km}$, respectively); $r_{c}$ is the classical radius of the electron, $2.8 \times 10^{-15}$ meter; and $\Delta N$ is the rms fluctuation in free-electron density. A value of $\Delta N=10^{11} /$ meter $^{3}$ is believed to be representative of the severe fluctuations encountered during periods of intense sunspot activity. Use of this value yields a phase fluctuation of 0.44 radian rms at $1000 \mathrm{mc}$. The ionospheric effect, from (7), varies inversely with the operating frequency. The desired relativistic effects, from (5), vary directly with frequency. Therefore, the fractional measurement error caused by ionospheric turbulence will vary inversely as the square of the operating frequency. Recent UHF measurements indicate that the phase fluctuation in propagation through the ionosphere is much less than one radian rms during a daytime period of normal ionospheric activity.

The Doppler-cancelling property of the two-way propagation experiment will effect cancellation of phase (or frequency) fluctuations resulting from propagation anomalies, as well as the radial Doppler shift resulting from the motion of the satellite, to the extent that correlation exists between the fluctuations introduced in the upward and downward traversals of the turbulent medium. The correlation coefficient is still to be estimated, in order to determine the residue of the calculated one-way phase fluctuation that will remain uncancelled in the experiment. It is known that the ionosphere rotates with the earth, and hence the upwardand downward-ray paths pass through essentially the same region of the ionosphere. The round-trip time delay, $\tau$, associated with the largest anticipated range $(10,000$ miles $)$ is 0.1 second. The value of the correlation coefficient for phase fluctuations displaced up to 0.1 second in time is therefore required. Experimental data $^{6,9}$ indicate maximum fluctuation rates of approxi- mately 4 cycles per minute, occurring during periods of magnetic activity. Assuming an exponential correlation function, we have

$$
\rho(\tau)=e^{-\omega_{0} r},
$$

where $\omega_{0}=2 \pi / 15=0.42 \mathrm{radian} / \mathrm{second}$.

The residual (uncancelled) phase error is then given by

$$
\Delta \psi_{\mathrm{rms}}=\psi_{\mathrm{rmss}}[2(1-\rho)]^{1 / 2}
$$

which, for small values of $\omega_{0} \tau$, is approximately

$$
\Delta \psi_{\mathrm{rms}} \approx \psi_{\mathrm{rms}}\left(2 \omega_{0} \tau\right)^{1 / 2}, \quad\left(\omega_{0} \tau \ll 1\right) .
$$

For $\tau=0.1$ second, the quantity $\left(2 \omega_{0} \tau\right)^{1 / 2}$ in $(10)$ is equal to $1 / 3.5$. The Doppler-cancellation process is therefore effective in reducing ionospheric phase fluctuations by a factor of 3.5 , or better. The uncancelled phase shift is found to be $7.4^{\circ} \mathrm{rms}$ at $1000 \mathrm{mc}$, or $15^{\circ} \mathrm{rms}$ at $500 \mathrm{mc}$, for a path length (one-way) of 10,000 miles. Even at the lower operating frequency, the expected error for a 60 second measurement interval is only $0.0014 \mathrm{cps}$, or 0.4 per cent of the expected maximum red shift.

We conclude that for operating frequencies in the range $500-1000 \mathrm{mc}$, propagation anomalies will produce a negligible deterioration in the accuracy of the measurement.

\section{APPENDiX}

We shall outline a method for computation of the Doppler frequency shift associated with the propagation of electromagnetic signals between points in nonuniform motion in a gravitational field. An approximate result is derived here; an exact derivation has been made by Friedman."

If a gravitational field in space is described in a system of coordinates $x_{1}, x_{2}, x_{3}, x_{4}$, where $x_{4}=c t$, and $t$ represents "coordinate time," then the relation

$$
d s^{2}=\sum_{\mu=1}^{4} \sum_{\nu=1}^{4} g_{\mu \nu} d x_{\mu} d x_{\nu}
$$

holds between each locally measured interval $d s$ and the corresponding coordinate differences, $d x_{\mu}$. For events occurring at a single point, the locally measured interval is given by

$$
d s=c d \tau,
$$

where $d \tau$ denotes "proper time" at that point. The quantities $g_{\mu \nu}$ define the metric properties of space (i.e., the gravitational field). For a spherically symmetric distribution of mass about the origin (Fig. 6), the $g_{\mu \nu}$ are given by the Schwarzschild solution to the field equations of general relativity:

${ }^{11}$ M. H. Friedman, Dept. of Physics, M.I.T., Cambridge, Mass., private communication; October, 1959. 


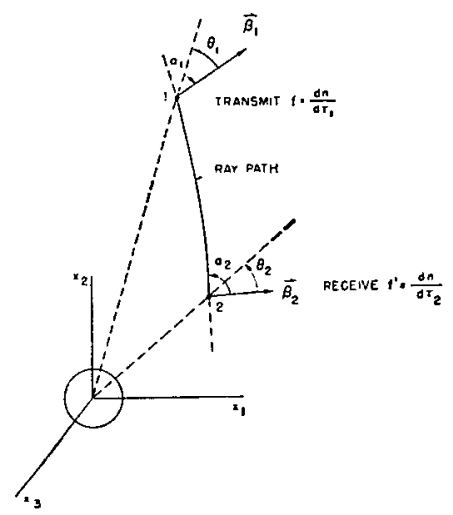

Fig. 6-Geometry of propagation between two points moving in a spherically symmetric gravitational field.

$$
\begin{aligned}
& g_{44}=1+2 \phi \\
& g_{4 \nu}=g_{\mu 4}=0 \\
& \left.g_{\mu \nu}=-\delta_{\mu \nu}+\frac{2 \phi}{1+2 \phi} \frac{x_{\mu} x_{\nu}}{r^{2}}\right\} \quad \begin{array}{l}
\mu=1,2,3 \\
\nu=1,2,3,
\end{array}
\end{aligned}
$$

where $\delta_{\mu \nu}=1(\mu=\nu) ; \delta_{\mu \nu}=0(\mu \neq \nu) ; r$ is the distance to the origin from the point $x_{1}, x_{2}, x_{3}$; and $\phi$ is the normalized gravitational potential at that point.

Consider a transmitter (Fig. 6) at point 1 in gravitational potential $\phi$, whose frequency $f$ is precisely controlled by a resonance of atoms, traveling with respect to the origin with (normalized) velocity $\beta_{1}$. The frequency transmitted may be regarded as some number of events per unit time. As viewed in the $x_{\mu}$ coordinate system, this frequency is given by

$$
f_{1}=\frac{d n}{d l_{1}}=\frac{d n}{d \tau_{1}} \frac{d \tau_{1}}{d l_{1}}=f \frac{d \tau_{1}}{d l_{1}} .
$$

Substitution for $g_{\mu \nu}$ and $d s$ in (11) yields

$$
\frac{d \tau_{1}}{d t_{1}}=\left[1+2 \phi_{1}-\beta_{1}^{2}\left(1-\epsilon_{1} \cos ^{2} \theta_{1}\right)\right]^{1 / 2},
$$

in which $\epsilon_{1}=\left(2 \phi_{1}\right) /\left(1+2 \phi_{1}\right) ; \theta_{1}$ is the angle between $\vec{\beta}_{1}$ and the radius to point 1 . The observer at point 2 is assumed to utilize a frequency standard of like constitu- tion to that at point 1 . By the same method, the frequency received at point 2 (viewed in the $x_{\mu}$ coordinate system) can be related to the frequency measured. Thus

$$
f_{2}=f^{\prime} \frac{d \tau_{2}}{d t_{2}}
$$

and

$$
\frac{d \tau_{2}}{d t_{2}}=\left[1+2 \phi_{2}-\beta_{2}{ }^{2}\left(1-\epsilon_{2} \cos ^{2} \theta_{2}\right)\right]^{1 / 2},
$$

where $d t_{2}$ is the (coordinate) time of arrival of the ray at point 2 . Since the number of events is preserved,

$$
d n=f^{\prime} \frac{d \tau_{2}}{d t_{2}} d t_{2}=f \frac{d \tau_{1}}{d t_{1}} d t_{1}
$$

Therefore

$$
f^{\prime}=f \frac{d t_{1}}{d t_{2}}\left[\frac{1+2 \phi_{1}-\beta_{1}{ }^{2}\left(1-\epsilon_{1} \cos ^{2} \theta_{1}\right)}{1+2 \phi_{2}-\beta_{2}{ }^{2}\left(1-\epsilon_{2} \cos ^{2} \theta_{2}\right)}\right]^{1 / 2} .
$$

The relationship between the coordinate times of reception and transmission is intractable because the ray velocity varies slightly along the path, and the path itself is curved. However, it can be shown that neglect of these effects leads to an error of the order of the satellite velocity multiplied by the gravitational potential, which is only approximately $10^{-14}$ for earth satellites. For present purposes, the derivation can be completed by a classical computation of the rate-of-change of path length:

$$
\frac{d t_{1}}{d l_{0}} \approx \frac{1-\beta_{2} \cos \alpha_{2}}{1+\beta_{1} \cos \alpha_{1}} .
$$

Moreover, $\epsilon_{1}$ and $\epsilon_{2}$ in (19) can be neglected. Thus,

$$
f^{\prime} \approx f\left[\frac{1+2 \phi_{1}-\beta_{1}{ }^{2}}{1+2 \phi_{2}-\beta_{2}{ }^{2}}\right]^{1 / 2}\left(\frac{1-\beta_{2} \cos \alpha_{2}}{1+\beta_{1} \cos \alpha_{1}}\right) .
$$

\section{ACKNOWLEDGMENT}

The authors wish to acknowledge the assistance given by Prof. M. H. Friedman, Prof. J. W. Graham, and V. J. Bates. They would also like to express their appreciation for the invaluable guidance of Prof. J. R. Zacharias. 